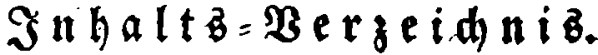

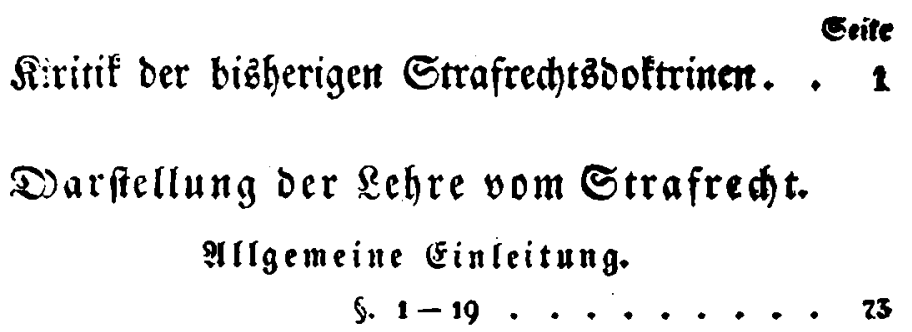

Darfellung ber Lehre felbif.

Zheoretifder Theil bez Etrafredtz.

Einleitung.

与. $20-22 \ldots ., \ldots 3$

\section{Erfte $\mathfrak{J} \mathfrak{a} \mathfrak{u} \mathfrak{p} \mathfrak{t} \mathfrak{t} \mathfrak{u} t$.}

Bom Snfalte ber Eontruttion oes Begriffo von

Strafbarfeit.

$$
\text { E res a a pitel. }
$$

Bon ber Matur ber hebertretung.

$$
\text { Erfier } \mathfrak{A} \mathfrak{h} \text { fhnitt. }
$$

Bon Der Jorm ber Lebertretung. 
3 weiter Hbidnitt.

Erite

Bom Snbalte ber Hebertretung.

5. $111-162$. . . . . . . 144

3 weites $R$ apitel.

Bon ber Ratur ber Etrafe.

Erfter Abfanitt.

Bom Snbalte Der Etrafe.

§. $163-195$. . . . . . . 186

3 weiter 2 bi由nitt.

\$on Der Gorw Der Strafe.

§. $196-215 . . . . . . .205$

3 roiteb $\mathfrak{S} a \mathfrak{u}$ tef

Bon ber Form ber Gonftrultion bez $\mathfrak{B e g r i f f B}$ vort

Strafbarteit.

Einteit ung.

๑. $216-219 . . .+. . .2 ?$

Eres sapitel.

Bon ber juriftifflen bermeneutif.

Erfer abidnitt.

Bon ber niedern Dermeneutit.

§. $220-24 \emptyset$. . . . . . . 223

3 weiter abiditt.

Bon Der böbern \$ermeneutif.

\$. $250-261 . . . . . .243$

3 weiteb anditer.

Bom rimterliकen Ermeffen.

§. $262-267 . . .+. . .257$ 
Wraltifder Ibeil bes Etrafredtz.

Grite

\section{Einleitung.}

§. $268 . . . . ., . .261$

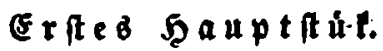

Qehre von ben Denumciationen.

Erftesapitel.

Eebre von ber Nielcuani.

§. $269-305$. . . . . . . . 262

3 weites sapitei.

Lebre von oer jurifiiden Bewiobeit.

5. $306-354$. . . . . 287

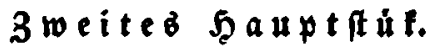

Sehre vom projeßs.

Erfes a a itel.

Bon oer innern Ratur bes geriळtliden Berfabrens.

Erfter $\mathscr{A}$ b

Bon ber innern Ratur bet geridtliden Berfabrens an fid.

Erfte Hotbeilung.

Sebre von Der Rechtelraft.

§. $355-368$. . . . . . 323

3 weite Abtbeilung.

Bon oen formen iuriftifder Bewisbeit.

\$. $369-381 . . . . . . .335$

Dritte entbeilung.

Iebre bon ber Eompetenf.

§. $382-396$. . . . . . . . 344 
3 weiter 4 b f n itt.

Bom Unterf́ied jwifhen polizeigeridtlimem Berfabren und peinlidem Projes und ben jur Eröffnung Ded Eejtern

erforderlianen Bedingungen.

§. $307-416$....... 35j

3 weiteb sapited.

Bon der äufern form des geridtliden \$erfabrens.

§. $417-419 . . . . . . . .371$

$\mathfrak{A}$ i $\mathfrak{b} \mathfrak{a} \mathfrak{n} \mathfrak{g}$

\$on Der Dollftelfung bes urtheils.

§. 420 ........ 373 